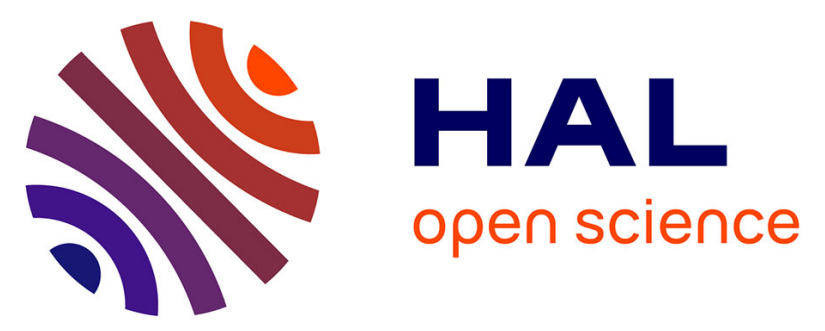

\title{
Phase resolved optical emission spectroscopy: a non-intrusive diagnostic to study electron dynamics in capacitive radio frequency discharges
}

J Schulze, E Schüngel, Z Donkó, D Luggenhölscher, U Czarnetzki

\section{- To cite this version:}

J Schulze, E Schüngel, Z Donkó, D Luggenhölscher, U Czarnetzki. Phase resolved optical emission spectroscopy: a non-intrusive diagnostic to study electron dynamics in capacitive radio frequency discharges. Journal of Physics D: Applied Physics, 2010, 43 (12), pp.124016. 10.1088/00223727/43/12/124016 . hal-00569562

\section{HAL Id: hal-00569562 https://hal.science/hal-00569562}

Submitted on 25 Feb 2011

HAL is a multi-disciplinary open access archive for the deposit and dissemination of scientific research documents, whether they are published or not. The documents may come from teaching and research institutions in France or abroad, or from public or private research centers.
L'archive ouverte pluridisciplinaire HAL, est destinée au dépôt et à la diffusion de documents scientifiques de niveau recherche, publiés ou non, émanant des établissements d'enseignement et de recherche français ou étrangers, des laboratoires publics ou privés. 


\title{
Phase Resolved Optical Emission Spectroscopy - A non-intrusive diagnostic to study electron dynamics in capacitive radio frequency discharges
}

\author{
J. Schulze ${ }^{1}$, E. Schüngel ${ }^{1}$, Z. Donkó ${ }^{2}$, D. Luggenhölscher ${ }^{1}$, and \\ U. Czarnetzki ${ }^{1}$ \\ ${ }^{1}$ Institute for Plasma and Atomic Physics, Ruhr-University Bochum, Germany \\ ${ }^{2}$ Research Institute for Solid State Physics and Optics of the Hungarian Academy of \\ Science, Budapest, Hungary \\ E-mail: fjschulze@hotmail.com
}

\begin{abstract}
Various types of capacitively coupled radio frequency (CCRF) discharges are frequently used for different applications ranging from chip and solar cell manufacturing to the creation of biocompatible surfaces. In many of these discharges electron heating and electron dynamics are not fully understood. A powerful diagnostic to study electron dynamics in CCRF discharges is Phase Resolved Optical Emission Spectroscopy (PROES). It is non-intrusive and provides access to the dynamics of highly energetic electrons, that sustain the discharge via ionization, with high spatial and temporal resolution within the RF period. Based on a time dependent model of the excitation dynamics of specifically chosen rare gas levels PROES provides access to plasma parameters such as the electron temperature, electron density and electron energy distribution function (EEDF). In this work the method of PROES is reviewed and some examples of its application are discussed. First, the generation of highly energetic electron beams by the expanding sheath in geometrically symmetric as well as asymmetric discharges and their effect on the EEDF are investigated. Second, the physical nature of the frequency coupling in dual frequency discharges operated at substantially different frequencies is discussed. Third, the generation of electric field reversals during sheath collapse in single and dual frequency discharges is analyzed. Then excitation dynamics in an electrically asymmetric novel type of dual frequency discharge is studied. Finally, limitations of PROES are discussed.
\end{abstract}

PACS numbers: 52.20.-j, 52.27.Aj, 52.40.Kh, 52.50.Qt, 52.70.-m, 52.70.Kz

Submitted to: J. Phys. D: Appl. Phys. 


\section{Introduction}

Capacitively coupled radio frequency $(\mathrm{CCRF})$ discharges are standard tools for many industrial applications such as etching and deposition processes [1]. Depending on their application various types of CCRF discharges operated under different conditions are used: Strongly geometrically asymmetric discharges are often used to generate a strong DC self bias to accelerate ions to high velocities perpendicular to the electrode in the sheath [1]. Dual frequency discharges operated at substantially different frequencies [2] and hybrid combinations of inductively and capacitively coupled discharges [3] are used to separately control the ion energy and the ion flux at the electrodes. Large area electrodes, e.g. $450 \mathrm{~mm}$ diameter, are required for next generation plasma reactors to process large area wafers [4]. Microscale atmospheric pressure plasma jets are used to provide high radical densities without the need of expensive vacuum systems [5].

Despite their enormous relevance for applications electron heating and electron dynamics in many of these discharge types are not understood. One reason for this is the difficulty to get access to the transient dynamics of highly energetic electrons experimentally with high enough spatial and temporal resolution of typically a few nanoseconds within the RF period. Phase Resolved Optical Emission Spectroscopy (PROES) provides this access non-intrusively using a simple experimental setup. Due to the thresholds for electron impact excitation of the observed levels PROES is only sensitive to highly energetic electrons (typically $E>10 \mathrm{eV}$ ). However, these electrons are particularly interesting, since they dominate the ionization and sustain the discharge. Based on a time dependent model of the excitation dynamics of specifically chosen rare gas levels it even provides space and time resolved access to plasma parameters such as the electron temperature, electron density and electron energy distribution function (EEDF) of highly energetic electrons. Furthermore, PROES provides a basis for measurements of quenching coefficients $[6,7]$ and RF-Modulation Spectroscopy (RFMOS) [8].

For the first time de Rosny et al. performed PROES measurements and found, that the electron impact excitation into specifically chosen atomic energy levels is time modulated within the RF period in a CCRF discharge operated at $13.56 \mathrm{MHz}[9]$. Other authors then found different excitation maxima within the RF period in different types of single frequency CCRF discharges caused by, among others, the expanding and collapsing sheath, secondary electrons, and heavy particle collisions [6,7,10-16]. Similar phenomena were observed using fluid and Particle in Cell (PIC) simulations. PIC simulations of Wood [17] and Vender et al. [18,19] predicted the generation of highly energetic electron beams by the expanding sheath, that propagate through the plasma bulk at low pressures, if the electron mean free path is long enough, and hit the opposing sheath, where they are reflected back into the discharge. Belenguer and Boeuf observed the transition from $\alpha$ - to $\gamma$-mode operation with increasing RF voltage at high pressures using a fluid simulation [20]. Recently, PROES measurements of the excitation dynamics of highly energetic electrons were extended to classical 
dual frequency discharges operated at substantially different frequencies [21-23] and micro plasmas $[24,25]$. Based on the method of trace rare gas optical emission spectroscopy [26,27] Gans et al. determined the electron temperature, the density of highly energetic electrons, and the EEDF space and time resolved via PROES in a geometrically asymmetric hydrogen discharge at high pressure [13]. Later, this method was applied to a classical dual frequency discharge to measure plasma parameters space and phase resolved [21]. PROES can also be used to study electron dynamics in other discharge types such as inductively coupled RF discharges [8, 28-30], where the temporal modulation of the emission within the RF period is typically much weaker compared to CCRF discharges.

In this work the basics of PROES are reviewed and some recent advances of the understanding of electron dynamics in different types of CCRF discharges are discussed. The paper is structured in the following way: In the second section a typical experimental setup for PROES measurements in CCRF discharges is described. In the third section the theory required to calculate the excitation from the measured spatiotemporal emission to eliminate the influence of the effective lifetime of the observed level is discussed. Additionally, the model to determine plasma parameters as well as the criteria for lines useful for PROES are introduced. In section 4 different applications of PROES are examined: The theoretically predicted generation of highly energetic electron beams by the expanding sheath and their reflection at the opposing plasma boundary is verified experimentally in different types of CCRF discharges. The physical nature of the frequency coupling in classical dual frequency discharges and the generation of field reversals during sheath collapse are understood based on PROES measurements. Furthermore, electron dynamics in a novel kind of electrically asymmetric, geometrically symmetric dual frequency discharge is studied and found to work differently compared to classical CCRF discharges. Then limitations of PROES are discussed. Finally, conclusions are drawn.

\section{Experimental setup}

A typical experimental setup used for PROES measurements in a CCRF discharge is shown in figure 1 . The plasma is ignited between two plane parallel electrodes of either the same or different surface areas inside a vacuum GEC chamber. An RF voltage waveform is applied to the bottom electrode through a matching unit. The discharge can be operated as a single, dual or multi-frequency discharge with phase locked harmonics.

The emission from a specifically chosen neon state $\left(N e 2 p_{1}\right)$ at $585.5 \mathrm{~nm}$ is measured space and phase resolved by a fast-gateable (gatewidth of a few ns) ICCD camera, e.g. Andor Istar, Roper PI-MAX, PicoStar LaVision, with a high repetition rate $(\geq 20 \mathrm{kHz})$ synchronized with the applied RF voltage waveform (see figure 2). In case of phase locked dual or multi frequency discharges the camera is synchronized with the lowest frequency RF voltage waveform. The camera's repetition rate is particularly important, since it limits the speed of each measurement by determining the number of consecutive 


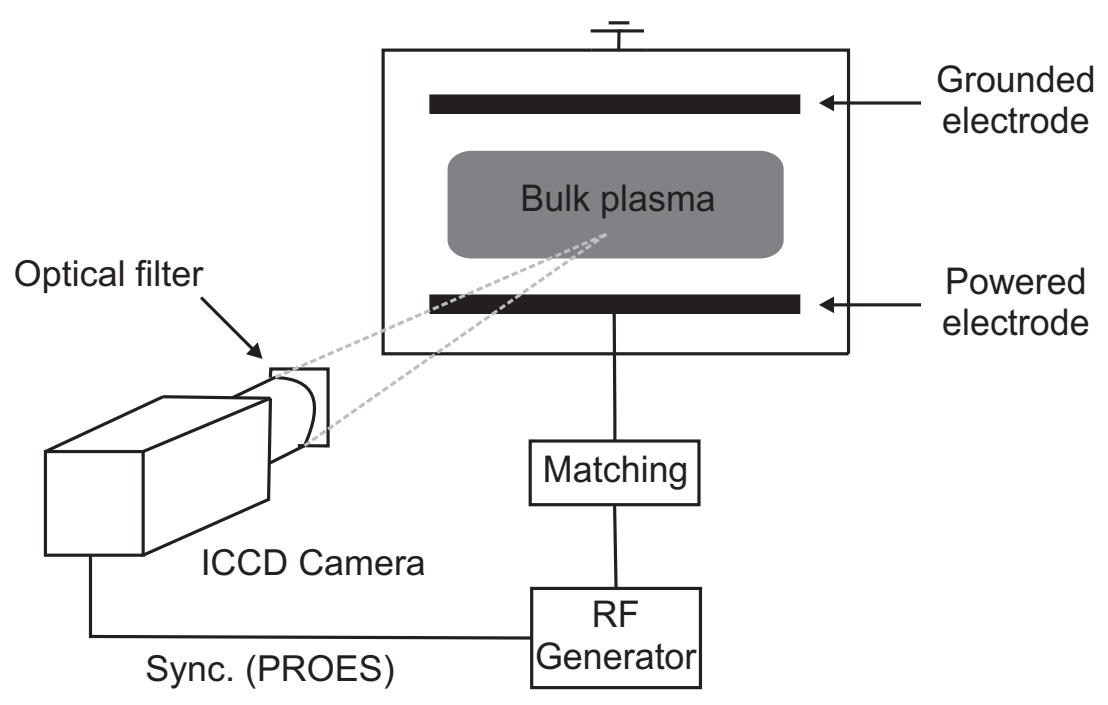

Figure 1. Typical experimental setup used for investigations of the excitation dynamics in a CCRF discharge by PROES.

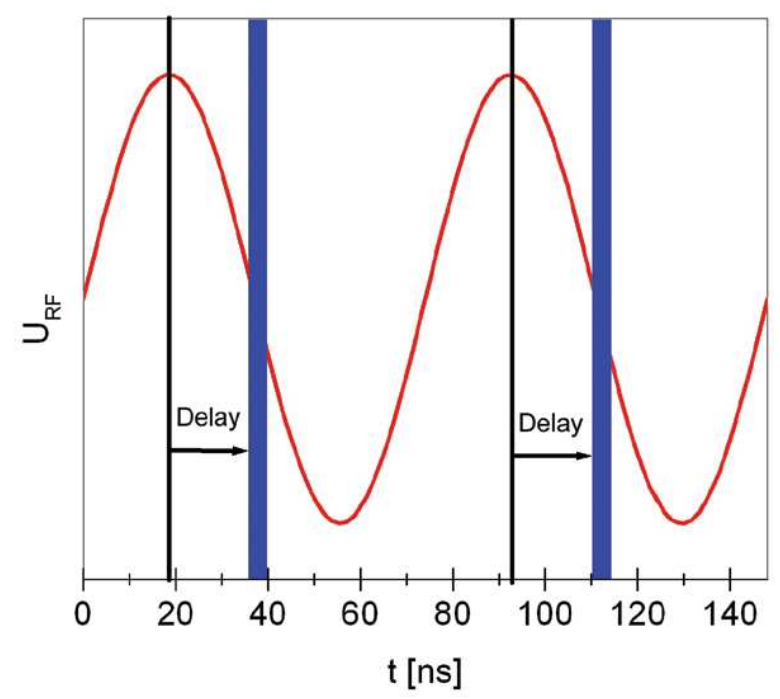

Figure 2. Principle of Phase Resolved Optical Emission Spectroscopy

$\mathrm{RF}$ periods that cannot be used for emission measurements. At repetition rates higher than the driving frequency every RF period can be used for emission measurements. The emission is observed through an adequate optical filter with two dimensional spatial resolution line integrated in the direction of the line of sight. In order to perform phase resolved measurements the delay generator of the ICCD camera sets a certain delay between the trigger and the camera gate (see figure 2). The signal is acquired at a certain phase during several thousands of RF periods. Then the delay is increased and the next phase is scanned. As only CCRF discharges with homogeneous emission 
parallel to the electrodes are investigated, all images are binned in horizontal direction in order to reduce the noise resulting in one dimensional spatial resolution along the discharge axis. Typically a temporal resolution of about $5 \mathrm{~ns}$ and a spatial resolution of about $0.5 \mathrm{~mm}$ are achieved. The temporal resolution is limited by the minimum gate width of the camera and the effective lifetime of the observed energy level.

Although the experimental setup is not always identical for the investigations discussed in this work the basic principle of PROES always remains the same.

\section{Theory}

From the measured spatio-temporal emission the electron impact excitation from the ground state $E_{0, i}(t)$ is calculated space and time resolved to eliminate the influence of the lifetime of the observed level. The population dynamics of an excited state $i$ with a population density $n_{i}$ is described by the following rate equation:

$$
\frac{d n_{i}(t)}{d t}=n_{0} E_{0, i}(t)+\sum_{m} n_{m} E_{m, i}(t)+\sum_{c} A_{c i} n_{c}(t)-A_{i} n_{i}(t)
$$

Here $n_{0}$ is the population density of the ground state. The term $n_{m} E_{m, i}(t)$ represents excitation out of metastable levels $m$ of population density $n_{m}$. In this context $E_{m, i}(t)$ is the electron impact excitation function for excitation from the metastable level $m$ into the observed level $i$. The term $A_{c i} n_{c}(t)$ describes additional population of level $i$ due to cascades from higher levels $c . n_{c}$ is the population density of the respective cascade level and $A_{c i}$ the decay rate for transitions from the cascade level $c$ into level $i$. $A_{i}$ is an effective decay rate, that takes into account reabsorption of radiation and radiationless collisional de-excitation, so called quenching:

$$
A_{i}=\frac{1}{\tau}=\sum_{k} A_{i k} g_{i k}+\sum_{q} k_{q} n_{q}
$$

Here $\tau$ is the effective lifetime of level $i$ and $A_{i k}$ is the transition probability of spontaneous emission from level $i$ to $k$. In equation 2 reabsorption of radiation is included by introducing escape factors $g_{i k}$, which reflect the probability of one photon originating from the transition from level $i$ to $k$ to leave the plasma without being reabsorbed. Reabsorption plays an important role in case of the overpopulated ground state. In comparison to other optically thin transitions, the effective transition rates into the ground state can be neglected $\left(g_{i k}=0\right)$. In general, reabsorption can reduce the effective decay rate or increase the effective lifetime of one state resulting in an apparent metastable state. Quenching is represented by the sum of all products of the density $n_{q}$ of all collision partners and the corresponding quenching coefficients $k_{q}$. In a de-excitation process, caused by quenching, energy is not lost by radiation, but is transferred into energy of both collision partners. Thus, quenching increases the effective decay rate $A_{i}$, reducing the lifetime and emission from a certain level $i$.

Equation 1 is part of a system of coupled differential equations comprising rate equations for all cascade and metastable levels. Furthermore, atomic and molecular data 
like quenching coefficients and decay rates need to be known. Thus, the determination of the electron impact excitation function $E_{0, i}(t)$ is generally difficult. In order to simplify equation 1 specific levels must be chosen, for which some population channels can be neglected.

The criteria that should be fulfilled are the following:

(i) low population due to cascades

(ii) low population due to excitation from metastable levels

(iii) knowledge of optical transition rates

(iv) enough intensity

(v) no superposition with other emission lines

(vi) short lifetime in order to temporally resolve the RF period (typically $74 \mathrm{~ns}$ )

(vii) influence of quenching is generally low

One energy level, that fulfills these criteria well, is $N e 2 p_{1}$. It has a short lifetime of only $14.5 \mathrm{~ns}$ [31], which allows access to excitation dynamics within the RF period of typically about $74 \mathrm{~ns}$. The threshold energy for electron impact excitation from the ground state is $19 \mathrm{eV}$. The contribution of cascades to the population of this level is particularly low [32].

Neglecting cascade contributions and excitation out of metastable states the rate of electron impact excitation from the ground state $E_{0, i}(t)$ is determined from the measured emission without any assumption of a particular shape of the EEDF [6]:

$$
E_{i, 0}(t)=\frac{1}{A_{i k} n_{0}}\left(\frac{d \dot{n}_{p h, i}(t)}{d t}+A_{i} \dot{n}_{p h, i}(t)\right)
$$

with

$$
\dot{n}_{p h, i}(t)=A_{i k} n_{i}(t)
$$

Here $\dot{n}_{p h, i}(t)$ is the measured number of photons per unit volume and time. $n_{0}$ is unknown and, therefore, only relative values of the excitation rate are calculated. Using equation 3 a spatio-temporal excitation matrix is calculated from the measured spatio-temporal emission matrix.

PROES is based on time dependent measurements of the population densities of specifically chosen excited rare gas states. A time dependent model, based on rate equations, describes the dynamics of the population densities of these levels. First order cascade contributions can be included [13,21]. Based on this model and the comparison of the excitation of several different rare gas states, with different excitation thresholds (trace rare gas optical emission spectroscopy) several plasma parameters can be determined space and phase resolved [26, 27].

$$
E_{i, 0}(t)=n_{e} \int_{0}^{\infty} \sigma_{i}(\epsilon) \sqrt{\frac{2 E}{m_{e}}} f_{e}(\epsilon) d \epsilon
$$


Here $\sigma_{i}(\epsilon)$ is the energy dependent electron impact excitation cross section for excitation from the ground state into level $i$ and $f_{e}(\epsilon)$ is the EEDF. If $E_{i, 0}(t)$ is known from this model for different energy levels, parameters such as the electron temperature, electron density as well as electron energy distribution functions of highly energetic electrons can be determined based on equation 5, which then corresponds to a set of equations for a certain number of unknowns (plasma parameters). For this analysis an adequate ansatz for the EEDF depending on the individual discharge must be made and the cross sections for electron impact excitation from the ground state must be accurately known for all energy levels involved. For rare gases such as argon, neon, krypton, and xenon these cross sections are provided by Lin et al. [32-36]. Small amounts of such rare gases are typically admixed as tracer gases for PROES, since they are little intrusive. The number of energy levels, that must be compared, depends on the number of plasma parameters to be determined, which in turn depends on the ansatz for the EEDF, e.g. Maxwellian EEDF, Bi-Maxwellian EEDF, etc. Details of this technique are described elsewhere $[13,21]$.

\section{Results}

\subsection{Electron beams generated by the expanding sheath}

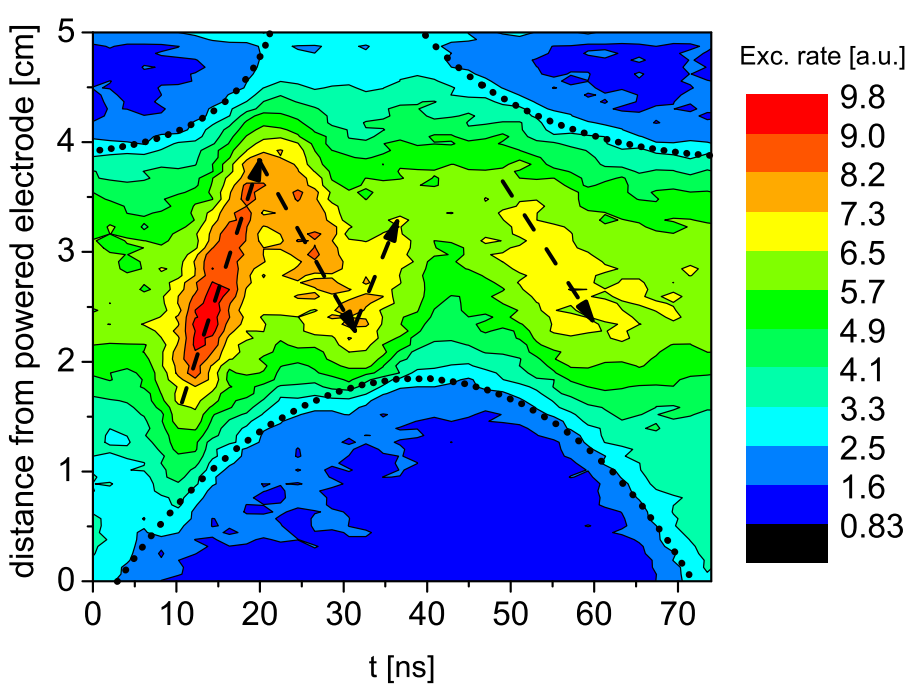

Figure 3. Spatio-temporal excitation into $N e 2 p_{1}$ in a single frequency discharge with equal electrode surface areas operated in neon at $13.56 \mathrm{MHz}, 2 \mathrm{~Pa}, 140 \mathrm{~W}$, and $\mathrm{d}=5$ $\mathrm{cm}$. The dotted lines indicate the sheath movement and the arrows indicate trajectories of highly energetic electron beams generated by the expanding sheath.

The generation of highly energetic electron beams by the expanding sheath and their reflection at the opposing modulated plasma boundary sheath predicted by PIC 
simulations of Wood [17] and Vender et al. $[18,19]$ is verified experimentally by PROES measurements. Figure 3 shows the spatio-temporal electron impact excitation rate from the ground state into $N e 2 p_{1}$ calculated from the measured spatio-temporal emission using equation 3 in a single frequency discharge with equal electrode surface areas operated in neon at $13.56 \mathrm{MHz}, 2 \mathrm{~Pa}, 140 \mathrm{~W}$, and an electrode gap of $\mathrm{d}=5 \mathrm{~cm}$. Essentially the experimental setup shown in figure 1 is used here with the difference that the plasma is shielded from the outer grounded chamber wall by a quartz cylinder to improve the geometrical symmetry. The bottom electrode is driven by only one frequency. The dotted lines in figure 3 indicate the sheath movement and the arrows indicate the trajectories of highly energetic electron beams generated by the expanding sheath. At the beginning of the RF period an electron beam is generated at the bottom electrode. It propagates through the entire bulk and hits the opposing collapsing sheath. The incoming beam is reflected back into the bulk. The reflected beam hits the fully expanded sheath at the bottom electrode and is reflected again until it is randomized by collisions after about one mean free path. This electron ping pong leads to an effective confinement of highly energetic electrons in the discharge. Due to the discharge symmetry another electron beam is generated by the expanding sheath at the top electrode. The excitation caused by this electron beam is weaker, since the discharge is not perfectly symmetric due to parasitic capacitive coupling between the glass cylinder confining the plasma and the outer grounded chamber wall [37]. These beam-like highly energetic electrons might not only gain energy from an acceleration by the expanding sheath, but also from the spatial profile of the plasma potential. This might cause the local extrema of the excitation at the discharge center.

A similar phenomenon is observed in geometrically strongly asymmetric discharges, where the surface area of the grounded electrode is much larger than the surface area of the powered electrode [38-40]. In this case an electron beam is only generated by the expanding sheath at the smaller powered electrode. Multiple reflections of the beam between an opposing floating surface and the modulated sheath adjacent to the powered electrode are observed. An important difference between symmetric and asymmetric CCRF discharges operated at low pressures is the presence of self-excited non-linear Plasma Series Resonance (PSR) oscillation of the RF current, which occur only in asymmetric discharges [40-44]. These PSR oscillation and Non-Linear Electron Resonance Heating cause a faster expansion of the sheath and, therefore, enhance the generation of highly energetic electron beams. A simple analytical model described in detail in reference [38] demonstrates that these highly energetic electron beams lead to an enhanced high energy tail of the EEDF (Bi-Maxwellian EEDF) and are, therefore, closely related to stochastic heating [45].

\subsection{Electron dynamics in classical dual frequency discharges}

PROES measurements in a classical symmetric dual frequency discharge $(1.937+27.118$ $\mathrm{MHz})$ operated in a gas mixture of helium $(72 \%)$ and $\mathrm{O}_{2}(19 \%)$ with a $9 \%$ admixture 


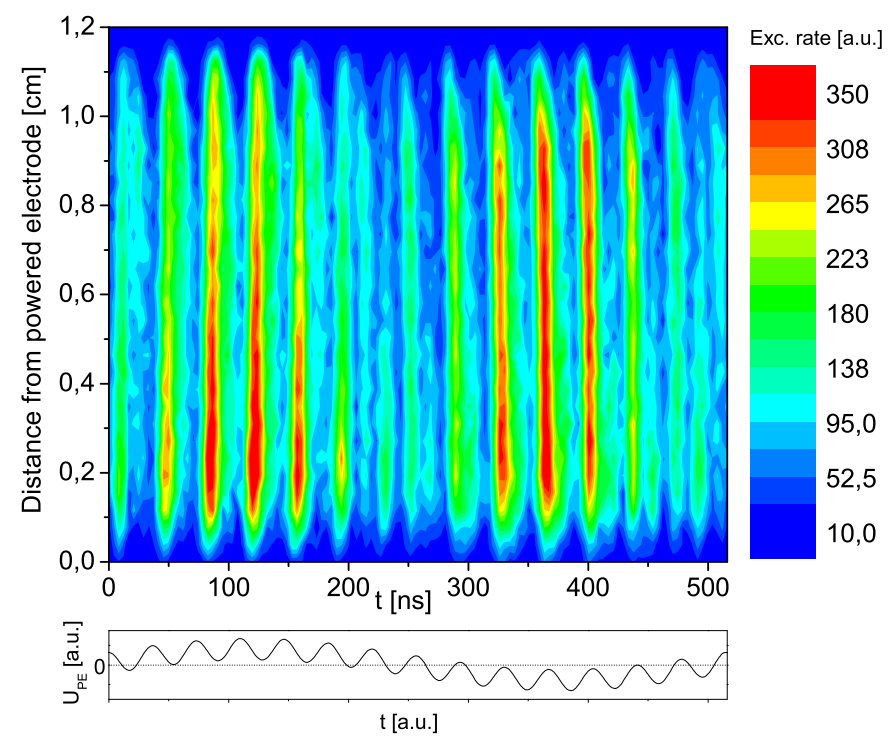

Figure 4. Spatio-temporal excitation into $N e 2 p_{1}$ in an industrial symmetric dual frequency discharge $(1.937+27.118 \mathrm{MHz})$ operated at $65 \mathrm{~Pa}$ and sketch of the voltage drop across the discharge $\left(P_{l f}=200 \mathrm{~W}, P_{h f}=800 \mathrm{~W}\right)[22,46,47]$.

of neon used as reference gas for PROES provide valuable insights into the physical nature of the frequency coupling in these discharges [21,22,46-48]. Figure 4 shows the measured spatio-temporal electron impact excitation rate from the ground state into $N e 2 p_{1}$ in such a discharge operated at $65 \mathrm{~Pa}$ with an electrode gap of $1.2 \mathrm{~cm}$ $[22,46,47]$. The experimental setup is described in detail elsewhere [22]. Measurements are performed with a temporal resolution of about $4 \mathrm{~ns}$ resolving the electron dynamics within the high frequency (hf) period. The spatio-temporal excitation profile is complex and a strong coupling of both frequencies is obvious. Beam-like highly energetic electrons are only generated by the expanding sheath at one electrode, if the sheath oscillates in a region of low ion density directly in front of the electrode. Via this mechanism the low frequency (lf) component affects the excitation/ionization by determining the position of sheath oscillation. Therefore, beam-like highly energetic electrons are only generated at the bottom electrode during the first half of one lf period and only at the top electrode during the second half. A PIC simulation verified these results [47]. Additionally, secondary electrons contribute to the excitation mainly leading to a modulation with twice the low frequency $[47,49]$. Finally, electric field reversals during sheath collapse affected by the frequency coupling are found to cause excitation, too [46]. This frequency coupling limits the separate control of ion flux and ion energy at the electrode surfaces [50].

\subsection{Field Reversals during sheath collapse}

If electrons cannot follow the collapsing sheath an electric field reversal is generated to enhance the electron loss to the electrode to ensure flux compensation of electrons and 


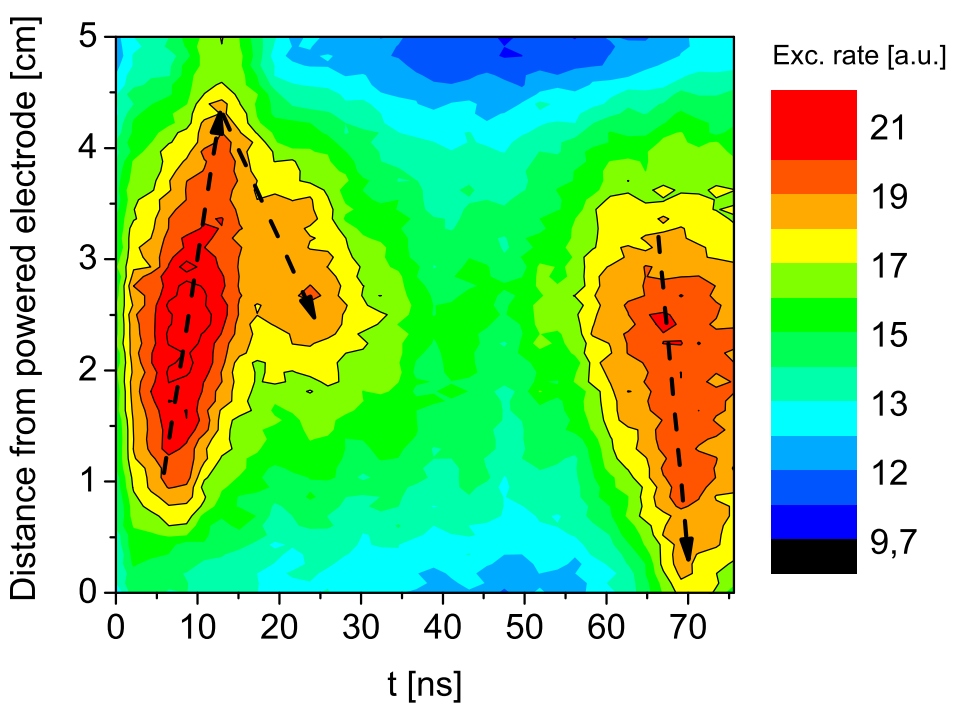

Figure 5. Spatio-temporal excitation into $N e 2 p_{1}$ in a strongly geometrically asymmetric single frequency $(13.56 \mathrm{MHz})$ neon discharge operated at $0.5 \mathrm{~Pa}, 8 \mathrm{~W}$ [46]. The powered electrode of smaller surface area and a floating surface (quartz) is located at $0 \mathrm{~cm}$ and $5 \mathrm{~cm}$, respectively.

ions at the electrode time averaged over one RF period. As electrons are accelerated towards the electrode by the reversed field, additional excitation can be observed by PROES during sheath collapse. Figure 5 shows the spatio-temporal electron impact excitation rate from the ground state into $N e 2 p_{1}$ in a strongly geometrically asymmetric single frequency $(13.56 \mathrm{MHz})$ neon discharge operated at $0.5 \mathrm{~Pa}$ and $8 \mathrm{~W}$ [46]. A quartz cylinder (floating surface) is located at a distance of $5 \mathrm{~cm}$ from the bottom powered electrode $(0 \mathrm{~cm})$. Again, the generation of a highly energetic electron beam during sheath expansion and its reflection at the quartz cylinder are observed at the beginning of the RF period. At the end of the RF period additional excitation caused by a field reversal is observed. This excitation maximum disappears with increasing pressure. The tilt of the corresponding electron trajectory indicates an acceleration of electrons towards the electrode. Under these low pressure conditions the field reversal is caused by electron inertia $[18,46,51]$ : Due to the low ion density the sheath is so big and collapses so fast, that electrons cannot follow the collapsing sheath due to their inertia. At high pressures field reversals can also occur, but are caused by collisions of electrons with the neutral background gas [12]. At high pressures theses electron-neutral collisions hinder electrons from following the sheath collapse and cause the field reversal. Generally, a low electron and high ion mobility are required for the generation of a field reversal. An analytical model explicitly shows that electron inertia and/or collisions of electrons with the neutral background gas can cause a field reversal $[12,46]$. In dual frequency discharges field reversals are also observed and caused by essentially the same mechanisms [46]. However, the frequency coupling affects their generation. 


\subsection{Excitation dynamics in electrically asymmetric discharges}
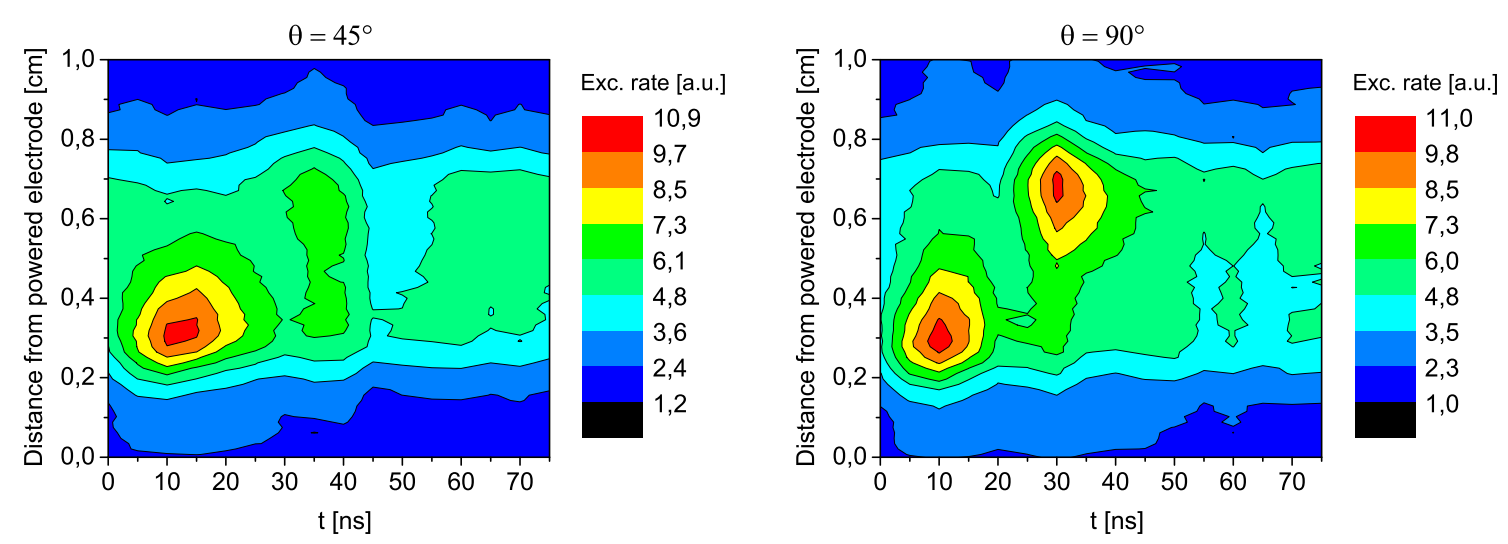

Figure 6. Spatio-temporal excitation into $N e 2 p_{1}$ in a geometrically symmetric dual frequency discharge driven at $13.56 \mathrm{MHz}$ and $27.12 \mathrm{MHz}$ with variable phase between the driving frequencies $\theta(74 \mathrm{~V}$ voltage amplitude of each applied voltage waveform, $60 \mathrm{~Pa}, 90 \%$ argon $+10 \%$ neon, and $\theta=45^{\circ}$ (left), $\theta=90^{\circ}$ (right)).

The Electrical Asymmetry Effect (EAE) [37, 44, 52-57] allows to generate a variable DC self bias even in geometrically symmetric dual frequency discharges driven at a fundamental and its second harmonic, e.g. $13.56 \mathrm{MHz}+27.12 \mathrm{MHz}$, with variable phase shift $\theta$ between the driving voltages. This $\mathrm{DC}$ self bias $\eta$ depends almost linearly on $\theta$ for $0^{\circ} \leq \theta \leq 90^{\circ}$. At about $0^{\circ}$ the DC self bias is stongly negative, at $45^{\circ}$ $\eta \approx 0 \mathrm{~V}$, and at $90^{\circ}$ it is strongly positive. Figure 6 shows the spatio-temporal electron impact excitation rate from the ground state into $N e 2 p_{1}$ in such a dual frequency discharge operated in argon with $10 \%$ admixture of neon at $60 \mathrm{~Pa}$ and $74 \mathrm{~V}$ voltage amplitude of each applied harmonic at $\theta=45^{\circ}$ (no bias) and $\theta=90^{\circ}$ (strongly positive bias). The excitation dynamics is dominated by sheath expansion heating and found to work substantially different compared to classical CCRF discharges: In classical CCRF discharges a DC self bias is generated via geometric asymmetry, i.e. different electrode surface areas. Thus, a strong bias will only be generated, if the electrode surface areas are significantly different. Assuming equal ion density profiles in front of both electrodes, this DC self bias will change the extrema of the sheath voltages and, therefore, the maximum sheath widths and expansion velocities in a way, that the sheath adjacent to the electrode of larger surface area is smaller compared to the sheath adjacent to the electrode of smaller surface area. Therefore, excitation due to sheath expansion heating will be stronger adjacent to the electrode of smaller surface area. Thus, in classical CCRF discharges a strong DC self bias will lead to spatio-temporal excitation profiles, that are asymmetric in the sense, that the maximum excitation at one electrode is stronger than at the other electrode. In electrically asymmetric geometrically symmetric discharges operated at high pressures this is substantially different (see figure 6): Here, the excitation is symmetric at a phase shift $\theta$ of strong DC self bias $\left(90^{\circ}\right)$ and strongly 
asymmetric at a phase shift of vanishing DC self bias $\left(45^{\circ}\right)$. This is caused by the fact, that the DC self bias is generated electrically instead of geometrically. In contrast to geometrically asymmetric discharges the bias ensures, that the absolute values of the extrema of the sheath voltages are identical at both electrodes. As can be demonstrated by an analytical model the electron drift velocity is determined only by the temporal derivative of the applied voltage waveform under these conditions. This model is based on the assumption of an electron velocity distribution function (EVDF) displaced by a drift velocity $u$ and an expansion of the EVDF with respect to $u$ to calculate the excitation rate. The drift velocity is calculated from the applied RF voltage waveform based on the voltage balance in the frame of a global model of an electrically asymmetric CCRF discharge. This model and the excitation dynamics will be discussed in detail in an upcoming publication.

\subsection{Limitations of PROES}

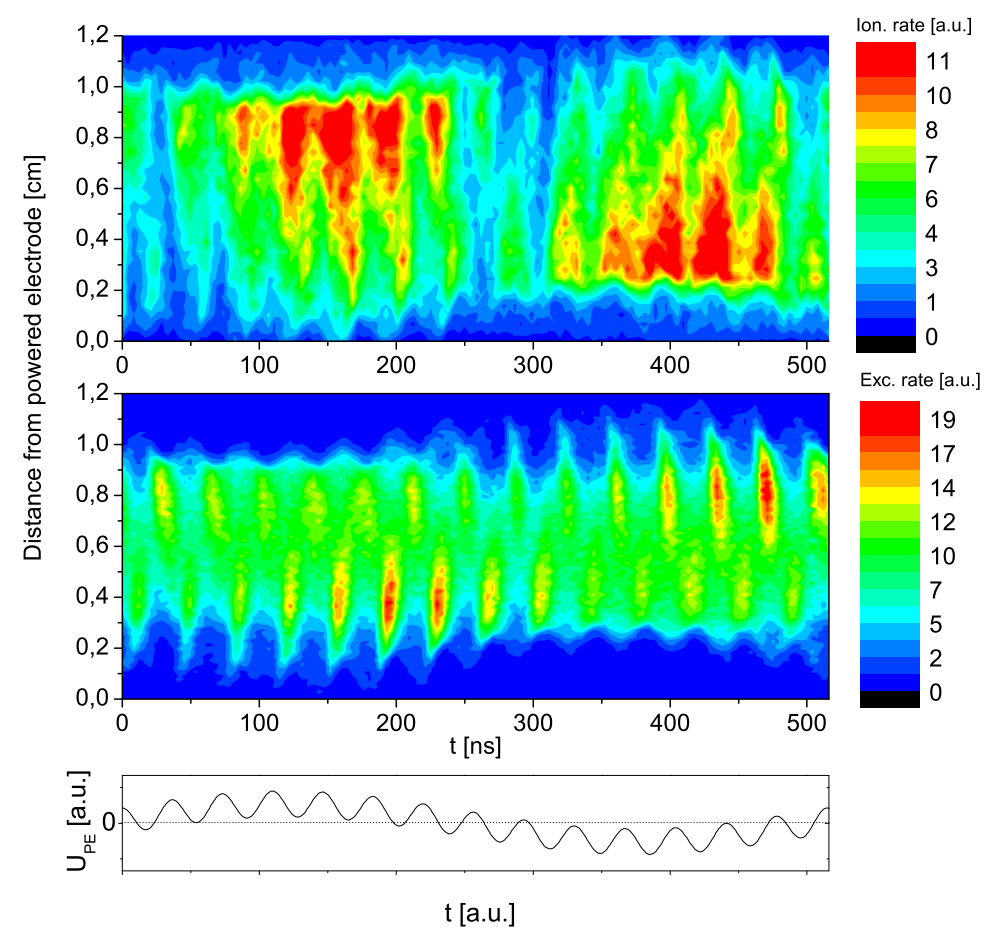

Figure 7. Total spatio-temporal ionization (top) and excitation (middle) obtained from a PIC simulation in a geometrically symmetric dual frequency $(1.937+27.118$ $\mathrm{MHz}$ ) argon discharge operated at $65 \mathrm{~Pa}$ [47] (500 V lf and $220 \mathrm{~V}$ hf amplitude of the applied voltage waveforms, $\gamma=0.1$ ). A sketch of the voltage drop across the discharge as a function of time within one lf period is also shown.

A fundamental limitation of PROES is its sensitivity to electrons within a certain energy range. This energy range is determined by the cross section for excitation into the observed level. Due to the threshold for excitation PROES is not sensitive to electrons below typically about $10 \mathrm{eV}$. Therefore, all plasma parameters determined by 
this diagnostic will be related to the high energy part of the EEDF only. PROES does not provide access to the majority of electrons at low energies. Thus, in order to get access to all electrons PROES must be combined with other diagnostics. However, the high energy part of the EEDF is particularly interesting for studies of electron heating and mechanisms of plasma sustainment.

At high electron energies another limitation might occur: Due to similarly shaped cross sections for excitation and ionization it is usually assumed that the ionization is probed by the excitation calculated from the emission measured by PROES. However, PIC simulation results of the spatio-temporal excitation and ionization show that this is not always the case (see figure 7). There can be significant differences between excitation and ionization, if secondary electrons contribute to the ionization [47], i.e. the ionization is dominated by secondary electrons, whereas the excitation is not. These secondary electrons can be so highly energetic (up to several hundred eV), that the cross section for ionization at these high energies is high, whereas the cross section for excitation is low [58], i.e. secondary electrons will cause ionization, however, they will hardly cause excitation and, therefore, might not be observed by PROES.

Another limitation of PROES is the fact, that only specifically chosen energy levels, that fulfill the requirements mentioned above under the relevant discharge conditions can be used for PROES, so that equation 3 can be used to calculate the excitation.

Finally, all quantities measured by PROES in the way described above are measured line integrated in the direction of the line of sight.

\section{Conclusions}

PROES is a powerful non-intrusive diagnostic, that provides access to highly energetic electrons with high spatial and temporal resolution on a nanosecond time scale within the RF period. Its experimental setup is particularly simple. Plasma parameters such as the electron density, electron temperature, and EEDF of highly energetic electrons can be measured. PROES provides the basis for measurements of quenching coefficients and RF-MOS. However, its limitations must be known.

PROES has provided valueable insights into electron heating in CCRF discharges: Beam-like highly energetic electrons accelerated by the expanding sheath and their reflection at the opposing plasma boundary at low enough neutral gas pressure is observed by PROES. Such electron beams lead to an enhanced high energy tail of the EEDF typical for stochastic heating. Electron dynamics and frequency coupling in classical dual frequency discharges operated at substantially different frequencies are understood based on PROES measurements. Electric field reversals are also traced by PROES and understood based on comparisons to simulations. Finally, electron dynamics in a novel kind of electrically asymmetric dual frequency CCRF discharge operated at a fundamental and its second harmonic with variable phase shift between the driving voltages is studied by PROES and found to work substantially different compared to classical CCRF discharges. 


\section{Acknowledgments}

This work has been funded by the DFG through GRK 1051, the Ruhr University Research School, and the Hungarian Scientific Research Fund through grant OTKAK-77653.

\section{References}

[1] Lieberman M A and Lichtenberg A J 2005, Principles of Plasma Discharges and Materials Processing, 2nd. ed., Wiley Interscience, NJ: Wiley

[2] Robiche J, Boyle P C, Turner M M, and Ellingboe A R 2003 J. Phys. D 361810

[3] Rauf S, and Kushner M J 1999 IEEE Trans. on Plasma Phys. 271329

[4] Chabert P 2007 J. Phys. D 40 R63

[5] Knake N, Niemi K, Reuter S, Schulz-von der Gathen V, and Winter J 2008 Appl. Phys. Lett. 93 131503

[6] Gans T, Lin C C, Schulz-von der Gathen V, and Döbele H F 2003 Phys. Rev. A 67012707

[7] Gans T, Lin C C, Schulz-von der Gathen V, and Döbele H F 2001 J. Phys. D 3439

[8] Crintea D L, Luggenhölscher D, Kadetov V A, Isenberg Ch., and Czarnetzki U 2008 J. Phys. D 41082003

[9] de Rosny G, Mosburg E R, Abelson J R, Devaud G, and Kerns R C 1983 J. of Appl. Phys. 54 2272

[10] Tochikubo F, Suzuki A, Kakuta S, Terazono Y, and Makabe T 1990 J. of Appl. Phys. 685532

[11] Flamm D L and Donnelly V M 1985 J. of Appl. Phys. 591052

[12] Czarnetzki U, Luggenhölscher D, and Döbele H F 1998 Plasma Sources Sci. and Techn. 8230

[13] Gans T, Schulz-von der Gathen V, and Döbele H F 1998 Europhys. Lett. 66232

[14] Nemschokmichal S, Dittmann K, and Meichsner J 2008 IEEE Trans. on Plasma Sci. 361360

[15] Dittmann K, Drozdov D, Krames B, and Meichsner J 2007 J. Phys. D 406593

[16] O'Connell D, Gans T, Vender D, Czarnetzki U, and Boswell R W 2008 Phys. of Plasmas 14034505

[17] Wood B P 1991 PhD thesis: Sheath Heating in Low Pressure Capacitive Radio Frequency Discharges University of California at Berkeley

[18] Vender D, and Boswell R W 1991 J. of Vac. Sci. Techn. A 101331

[19] Vender D, and Boswell R W 1990 IEEE Trans. on Plasma Sci. 18725

[20] Belenguer P, and Boeuf J P 1990 Phys. Rev. A 414447

[21] Gans T, Schulze J, O'Connell D, Czarnetzki U, Faulkner R, Ellingboe A R, and Turner M M 2006 Appl. Phys. Lett. 89261502

[22] Schulze J, Gans T, O'Connell D, Czarnetzki U, Ellingboe A R, and Turner M M 2007 J. Phys. D 407008

[23] O'Connell D, Gans T, Semmler E, and Awakowicz P 2008 Appl. Phys. Lett. 93081502

[24] Schulz von der Gathen V, Schaper L, Knake N, Reuter S, Niemi K, Gans T, and Winter J 2008 J. Phys. D 41194004

[25] Liu D W, Iza F, and Kong M G 2008 Appl. Phys. Lett. 93261503

[26] Malyshev M V and Donnelly V M 1997 J. Vac. Sci. Technol. A 15550

[27] Malyshev M V and Donnelly V M 1997 Phys. Rev. E 606016

[28] Czarnetzki U, Kadetov V A 2003 Proc. 56th Gaseous Electronics Conference, San Francisco, USA

[29] Gans T, Osiac M, O'Connell D, Kadetov V A, Czarnetzki U, Schwarz-Selinger T, Halfmann H, and Awakowicz P 2005 Plasma Phys. and Contr. Fus. 47 A353

[30] Kampschulte T, Schulze J, Gans T, Czarnetzki U, Marke S, and Wallendorf T 2005 Surf. Coat. Technol. 200859

[31] NIST atomic spectra database http: \\physlab.nist.gov $\backslash$ cgi-bin $\backslash$ AtData $\backslash$ main_asd.

[32] Chilton J E, Stewart M D, and Lin C C 2000 Phys. Rev. A 6152708 
[33] Chilton J E, Boffard J B, Schappe R S, and Lin C C 1998 Phys. Rev. A 57267

[34] Chilton J E and Lin C C 1999 Phys. Rev. A 603712

[35] Chilton J E, Stewart M D, and Lin C C 2000 Phys. Rev. A 6232714

[36] Fons J T and Lin C C 1998 Phys. Rev. A 584603

[37] Schulze J, Schüngel E, and Czarnetzki U 2009 J. Phys. D 42092005

[38] Schulze J, Heil B G, Luggenhölscher D, Mussenbrock T, Brinkmann R P, and Czarnetzki U 2008 J. Phys. D 41042003

[39] Schulze J, Heil B G, Luggenhölscher D, and Czarnetzki U 2008 IEEE Trans. on Plasma Sci. 361400

[40] Schulze J, Heil B G, Luggenhölscher D, Brinkmann R P, and Czarnetzki U 2008 J. Phys. D 41195212

[41] Czarnetzki U, Mussenbrock T, and Brinkmann R P 2006 Phys. of Plasmas 13123503

[42] Mussenbrock T, and Brinkmann R P 2006 Appl. Phys. Lett. 88151503

[43] Mussenbrock T, Brinkmann R P, Lieberman M A, Lichtenberg A J, and Kawamura E 2008 Phys. Rev. Lett. 101085004

[44] Donkó Z, Schulze J, Czarnetzki U, and Luggenhölscher D 2009 Appl. Phys. Lett. 94 131501

[45] Godyak V A, Piejak R B, and Alexandrovich B M 1992 Plasma Sources Sci. and Techn. 136

[46] Schulze J, Donkó Z, Heil B G, Luggenhölscher D, Mussenbrock T, Brinkmann R P, and Czarnetzki U 2008 J. Phys. D 41105214

[47] Schulze J, Donkó Z, Luggenhölscher D, and Czarnetzki U 2009 Plasma Sources Sci. and Techn. 18034011

[48] Turner M M and Chabert P 2006 Phys. Rev. Lett. 96205001

[49] Turner M M, Chabert P, Levif P, Boyle P, and Robiche J 2007 Conference Proceedings of 18. ICPIG, Prague

[50] Donkó Z, Petrovic Z L 2006 Jpn. J. Appl. Phys. Part 1458151

[51] Sato A H and Lieberman M A 1990 J. Appl. Phys. 68126117

[52] Heil B G, Schulze J, Mussenbrock T, Brinkmann R P, and Czarnetzki U 2008 IEEE Trans. on Plasma Sci. 361404

[53] Heil B G, Czarnetzki U, Brinkmann R P, and Mussenbrock T 2008 J. Phys. D 41165202

[54] Donkó Z, Schulze J, Heil B G, and Czarnetzki U 2008 J. Phys. D 42025205

[55] Czarnetzki U, Heil B G, Schulze J, Donkó Z, Mussenbrock T, and Brinkmann R P 2009 IOP Conf. Series 162012010

[56] Schulze J, Schüngel E, Donkó Z, and Czarnetzki U 2009 J. Appl. Phys. 106063307

[57] Longo S, and Diomede P 2009 Plasma Processes and Polymers 6370

[58] Phelps A V and Petrović Z Lj, 1999 Plasma Sources Sci. Technol. 8 R21 